\title{
Trocas interacionais no estudo de gêneros: focalizando o gênero profissional résumé
}

\author{
Ana Valéria Bisetto Bork' (UTFPR)
}

\begin{abstract}
Resumo: O presente artigo objetiva apresentar parte dos resultados de um estudo sobre o gênero profissional résumé, em que discussões ocorridas ao longo de um curso de escrita acadêmica são apontadas como responsáveis pela apropriação do gênero em estudo. Os construtos teóricometodológicos que fundamentam este estudo são o Interacionismo Social (VIGOTSKI, 2002), o Interacionismo Sociodiscursivo (BRONCKART, 2012), as capacidades de linguagem (CRISTOVÃO; STUTZ, 2011; BRONCKART, 2012) e o estudo de gêneros (SCHNEUWLY; DOLZ, 2004). A análise das interações e intervenções orais realizadas tem como base as categorias dos modos de participação dos interlocutores na interação em eventos de comentários sobre textos em progresso (GARCEZ, 2010). Com base nos dados de pesquisa, apresentamos uma expansão das categorias com uma descrição de andaimes identificados nas trocas interacionais.
\end{abstract}

Palavras-chave: Perspectiva sociocultural. Interação e mediação. Gênero profissional résumé.

\begin{abstract}
This article aims to present part of the results of a study on the professional genre résumé, in which discussions carried out throughout a course on academic writing are pointed out as responsible for the appropriation of the genre under study. The theoretical-methodological constructs which underlie this study are the Social Interactionism (VIGOTSKI, 2002), the Sociodiscursive Interactionism (BRONCKART, 2012), the language capacities (CRISTOVÃO; STUTZ, 2011; BRONCKART, 2012), and the study of genres (SCHNEUWLY; DOLZ, 2004). The analysis of oral interactions and interventions are based on the categories of the interlocutors' forms of participation in the interaction of comment events about texts in progress (GARCEZ, 2010). Based on the research data we present an expansion of the categories with a description of the scaffolds identified in the interactional exchanges.
\end{abstract}

Keywords: Sociocultural perspective. Interaction and mediation.

Professional genre résumé. 


\section{Introdução}

Ao nos relacionarmos com outras pessoas ao longo da vida, nos desenvolvemos e vamos construindo nossas identidades e saberes, os quais vão se transformando à medida que nos comunicamos e interagimos com o outro. É uma aprendizagem contínua e incessante, pois, segundo Leffa (2003, p. 2), "ninguém aprende sozinho, como também ninguém cresce, vive, sofre ou morre sozinho; estamos sempre agindo e reagindo com o contexto que nos cerca". Assim, a convivência nos diferentes grupos sociais faz com que passemos por momentos em que a ajuda do outro se torna crucial para a compreensão e realização de diferentes atividades, como a explicação de regras para um jogo coletivo ou a resolução de um problema.

Estudos realizados no campo da Linguística Aplicada (FIGUEIREDO, 2003; PAIVA, 2003; MOITA LOPES, 2006) reforçam a ideia de que devemos pensar sobre o ensino de línguas a partir das interações interpessoais. Logo, para a realização deste trabalho de pesquisa, utilizamos os aportes teóricos do interacionismo social de Vigotski (2002), representado pela noção de zona de desenvolvimento proximal (ZDP), e do conceito de andaime coletivo (DONATO, 1994). Além disso, o estudo está pautado no Interacionismo Sociodiscursivo (BRONCKART, 2012), nas capacidades de linguagem (CRISTOVÃO; STUTZ, 2011; BRONCKART, 2012) e no estudo de gêneros (SCHNEUWLY; DOLZ, 2004) como mediadores do processo interacional.

\section{Fatores mediação e interação}

A questão da mediação é vista como ponto fundamental no trabalho de Vigotski (2002), pois está sempre presente na atividade humana; é uma forma especializada de interação entre o sujeito que aprende e o sujeito que ensina. Como elementos básicos responsáveis pela mediação, o educador e psicólogo russo aponta os conceitos de instrumento e signo (instrumentos psicológicos), em que o primeiro tem o papel de regular os objetos, e o segundo tem a função de regular as ações sobre o psiquismo das pessoas.

A função do instrumento é servir como um condutor da influência humana sobre o objeto da atividade; ele é orientado externamente; deve necessariamente levar a mudanças nos objetos. Constitui um meio pelo qual a atividade humana é dirigida para o controle e domínio da natureza. 
O signo, por outro lado, não modifica em nada o objeto da operação psicológica. Constitui um meio da atividade interna dirigido para o controle do próprio indivíduo; o signo ${ }^{1}$ é orientado internamente. Essas atividades são tão diferentes uma da outra, que a natureza dos meios por elas utilizados não pode ser a mesma (VIGOTSKI, 2002, 72-73). (Grifos do autor)

Sobre as questões de mediação, utilizamos a metáfora do andaime (em inglês, scaffolding), criada para designar outro conceito relacionado à ajuda que o aprendiz recebe de outras pessoas que, segundo Wood, Bruner e Ross (1976, p. 90), "habilita uma criança ou um aprendiz a resolver um problema, a executar uma tarefa ou alcançar um objetivo que estaria além de seus esforços se não houvesse uma assistência". A função do par mais competente, denominado por Vigotski (2002) de mediador, é encontrar meios de ensinar o outro a aprender. A interação de pessoas mais experientes com outras menos experientes está vinculada ao conceito de internalização, processo de desenvolvimento que consiste em uma sequência de operações, o qual leva à transformação do processo interpessoal em intrapessoal. Ao pensar no desenvolvimento da criança, Vigotski (2008) aponta que todas as funções psíquicas aparecem duas vezes no ciclo do desenvolvimento humano: primeiro, no nível social, como uma atividade coletiva realizada a partir da mediação de outras pessoas ou de artefatos culturais (interpsicológica), e, posteriormente, no nível individual, como propriedade interior do pensamento da criança (intrapsicológica), a qual reconstrói recursos para regular suas próprias atividades. Com respeito à aprendizagem infantil, o papel do mediador pode ser ocupado pelos pais ou responsáveis, enquanto que no contexto educacional, a mediação pode ocorrer com a presença do professor ou dos colegas de classe, os quais podem auxiliar a criança nas etapas mais complexas do processo de aprendizagem.

Com relação às interações orais estabelecidas no ambiente escolar ${ }^{2}$, a mediação pode acontecer de forma dialogada, ou em pares, como a correção de um texto elaborado por um dos alunos; pode, ainda, se realizar de forma coletiva, por meio da colaboração de todos os envolvidos na execução de uma tarefa. Salientamos que a mediação não ocorre somente por meio da presença do outro, mas pode acontecer em razão da utilização de

\footnotetext{
${ }^{1}$ Dentre os signos listados por Vigotski (2002) que podem se tornar instrumentos psicológicos estão a linguagem, as formas de contagem e cálculo, os meios mnemotécnicos, as obras de arte, os esquemas e diagramas, entre outros.

${ }^{2}$ Sobre a distribuição de turnos em sala de aula, Cazden (1988) aponta que a estrutura interacional triádica mais utilizada pelos professores é: iniciação do professor, resposta do aluno e avaliação do professor (IRA).
} 
vários instrumentos que se encontram disponíveis no contexto de sala de aula, os quais se tornam também responsáveis pelo desenvolvimento do aprendiz.

Por acreditar no papel ativo do discente nas interlocuções que se realizam no contexto educacional, tomamos a concepção sociointeracional de ensino como o princípio norteador de nossa pesquisa, em que a linguagem é vista como prática social. É por este motivo que compreendemos a interação no cenário educacional como um momento de coconstrução de significados em que todos os participantes trabalham coletivamente e colaborativamente na construção do conhecimento, seja nas conversas e discussões entre professor-aluno, professor-alunos ou alunos-alunos. Ela é parte constitutiva da sala de aula, em que ao assumirem diferentes papéis na construção do conhecimento, todos se tornam mediadores no processo de ensino e aprendizagem.

Além do fator mediação, outra contribuição da psicologia vigotskiana é a proposição de que o pensamento humano deve ser compreendido em suas circunstâncias sociais e históricas. Logo, confere enorme importância ao papel da interação social no desenvolvimento do ser humano. Nas palavras de Rego (1995),

Vygotski afirma que as características tipicamente humanas não estão presentes desde o nascimento do indivíduo, nem são mero resultados das pressões do meio externo. Elas resultam da interação dialética do homem e seu meio sócio-cultural. Ao mesmo tempo em que o ser humano transforma o seu meio para atender suas necessidades básicas, transforma-se a si mesmo. (REGO, 1995, p.41)

Ao estudar o desenvolvimento infantil, não é possivel negar a importância das características biológicas no desenvolvimento e na evolução da criança, porém Vigotski salienta que o fator primordial está no papel da interação social. Deste modo, o homem constitui-se como tal por meio de suas interações sociais e é visto como alguém que transforma e é transformado nas relações produzidas em um determinado contexto.

Com o objetivo de materializar nossos pensamentos, ideias e sentimentos, utilizamos diferentes linguagens para nos comunicar com as pessoas. Para Vigotski (2002), a linguagem tem o papel central enquanto elemento mediador dos processos de construção das identidades sociais. Assim, a fala humana é considerada um dos mais importantes signos utilizados para a comunicação. O psicólogo russo afirma que 
[...] a linguagem, o próprio meio através do qual a reflexão e a elaboração da experiência ocorrem, é um processo extremamente pessoal e, ao mesmo tempo, um processo profundamente social. [...] A fala também é um uso excelente do uso de signos, já que, uma vez internalizada, torna-se uma parte profunda e constante dos processos psicológicos superiores ${ }^{3}$. (VIGOTSKI, 2002, p. 169)

Sobre questões relacionadas à capacidade de aprendizagem infantil, Vigotski (2002) identificou dois níveis de desenvolvimento: o nível de desenvolvimento real e o nível de desenvolvimento potencial. No primeiro caso, ele está relacionado ao "nível de desenvolvimento das funções mentais da criança que se estabeleceram como resultado de certos ciclos de desenvolvimento já completados" (VIGOTSKI, 2002, p.111), ou seja, concerne às conquistas já efetivadas pela criança, aquelas que ela consegue realizar sozinha. Já para o nível de desenvolvimento potencial, alusivo às capacidades em vias de serem construídas, a ajuda do outro é fundamental, seja pela presença de adultos ou de crianças mais experientes. Essa orientação ou colaboração com pares mais capazes faz com que as crianças alcancem níveis diferenciados de aprendizagem. Assim, Vigotski (2002, p. 112) define o conceito de zona de desenvolvimento proximal (ZDP) como

[...] a distância entre o nível de desenvolvimento real, que se costuma determinar através da solução independente de problemas, e o nível de desenvolvimento potencial, determinado através da solução de problemas sob a orientação de um adulto ou em colaboração com companheiros mais capazes.

Há, portanto, um despertar de procedimentos internos de desenvolvimento que não seriam possíveis de acontecer se não fossem possibilitados pelas relações que estabelecemos com os outros.

Tendo em vista as inúmeras possibilidades de interação e mediação que emergem em um contexto interativo de aprendizagem, várias ZDP podem ser criadas e conceitos novos, antigos ou cristalizados podem ser constantemente confrontados e compartilhados pelos participantes. Por meio das práticas discursivas presentes nas diversas situações de convívio social a que somos expostos, as críticas construtivas, as trocas de ideias, assim

\footnotetext{
${ }^{3}$ Vigoski explica que as funções psicológicas superiores estão relacionadas ao que é caracteristicamente humano, como o pensamento deliberado, a linguagem, a atenção, a memória voluntária, a escrita, entre outros, enquanto que as funções psicológicas elementares se referem ao que é biológico, as quais se fazem presentes desde os momentos iniciais do desenvolvimento humano, como os instintos e os reflexos, por exemplo.
} 
como os questionamentos, as discussões, as contradições e os conflitos são vistos aqui como pontos positivos para o processo de desenvolvimento e aprendizagem do indivíduo.

Além do conceito da ZDP, Vigotski (2008) apresenta a distinção entre conceitos cotidianos (ou espontâneos) e científicos. No caso dos conceitos cotidianos, estes se referem às atividades concretas vivenciadas em contextos sociais nos quais o ser humano se desenvolve, sem necessitar de um "quadro institucional, pois a criança os forma e os aprende em sua atividade prática, na comunicação imediata com as pessoas, [...] nas situações informais de aprendizagem" (FRIEDRICH, 2012, p. 99). Sobre os conceitos científicos, estes resultam de uma investigação teórica e são sempre mediados por outros conceitos, permitindo uma mudança na relação cognitiva do homem com o mundo.

Ao pensarmos no estudo de uma língua estrangeira (LE), esta se vincula ao conhecimento científico por sua apreensão acontecer de forma deliberada em um contexto formal de aprendizagem, diferentemente do que ocorre com a língua materna (LM), a qual é apreendida espontaneamente. Sobre essa questão, Vigotski (2008) atenta que

O êxito no aprendizado de uma língua estrangeira depende de um certo grau de maturidade da língua materna. A criança pode transferir para a nova língua o sistema de significados que já possui na sua própria. $O$ oposto também é verdadeiro - uma língua estrangeira facilita o domínio das formas mais elevadas da língua materna. [...] Não é de surpreender que exista uma analogia entre a interação das línguas materna e estrangeira e a interação dos conceitos científicos e espontâneos, já que ambos os processos pertencem à esfera de desenvolvimento do pensamento verbal (VIGOTSKI, 2008, p. 137).

Ao desenvolver o conceito de ZDP e dedicar seus estudos às questões de interação e mediação, Vigotski nos fornece elementos importantes para compreender como se dá a integração entre as questões de ensino e aprendizagem e o processo de desenvolvimento no contexto educacional.

\section{O gênero résumé}

O gênero résumé focaliza as realizações de cunho profissional e acadêmico de uma pessoa. As informações não se encontram descritas em forma de um texto narrativo, mas de modo topicalizado, em que os itens mencionados pelo agente produtor aparecem separadamente. Pode, assim, ser definido como um documento utilizado por pessoas que 
desejam apresentar um relato breve e direto sobre sua vida profissional e acadêmica, cujo objetivo está em informar o leitor sobre as principais atividades profissionais e acadêmicas realizadas pelo sujeito que deseja entrar no mundo do trabalho, ou ainda buscar um novo emprego. Assim, no momento da organização do résumé, o candidato deve mencionar seu histórico profissional, suas habilidades e conquistas relacionadas à vaga preterida.

Considerando o contexto brasileiro, seu estilo composicional apresenta traços semelhantes ao do gênero curriculum vitae $(\mathrm{CV})$ e, até mesmo, do currículo Lattes ${ }^{5}$, compartilhado no meio virtual e utilizado por alunos, professores e pesquisadores que postam informações sobre suas realizações acadêmicas e profissionais.

Whitcomb (2010) apresenta quatro diferentes tipos de résumé, independentemente da área profissional pretendida. As informações do candidato podem ser postadas na forma de um résumé de ordem cronológica, funcional, de combinação, ou no formato de résumé direcionado. Segundo a autora, o résumé cronológico é o tipo mais utilizado e o preferido dos empregadores, justamente por mostrar informações de forma clara, podendo incluir o objetivo ou um resumo sobre a carreira pretendida; faz uma apresentação de dados históricos e profissionais, partindo das posições mais recentes ocupadas pelo candidato. Os tópicos alusivos à educação, certificações e habilidades são listados após o item experiência.

Trazemos, a seguir, um exemplo de résumé cronológico, referente à posição de repositor de roupas (retail position), retirado de um site relacionado à área profissional.

\footnotetext{
${ }^{4} \mathrm{O}$ curriculum vitae é um documento escrito no qual constam os principais dados pessoais de uma pessoa, o resumo de sua vida escolar e acadêmica e informações alusivas à sua experiência profissional. Essa expressão latina significa, literalmente, resumo da vida.

${ }^{5}$ A Plataforma Lattes, criada e mantida pelo Conselho Nacional de Desenvolvimento Científico e Tecnológico (CNPq), integra as bases de dados de currículos, grupos de pesquisa e instituições, em um único sistema de informações, das áreas de Ciência e Tecnologia no Brasil.
} 
Quadro 1 - Texto de referência: résumé

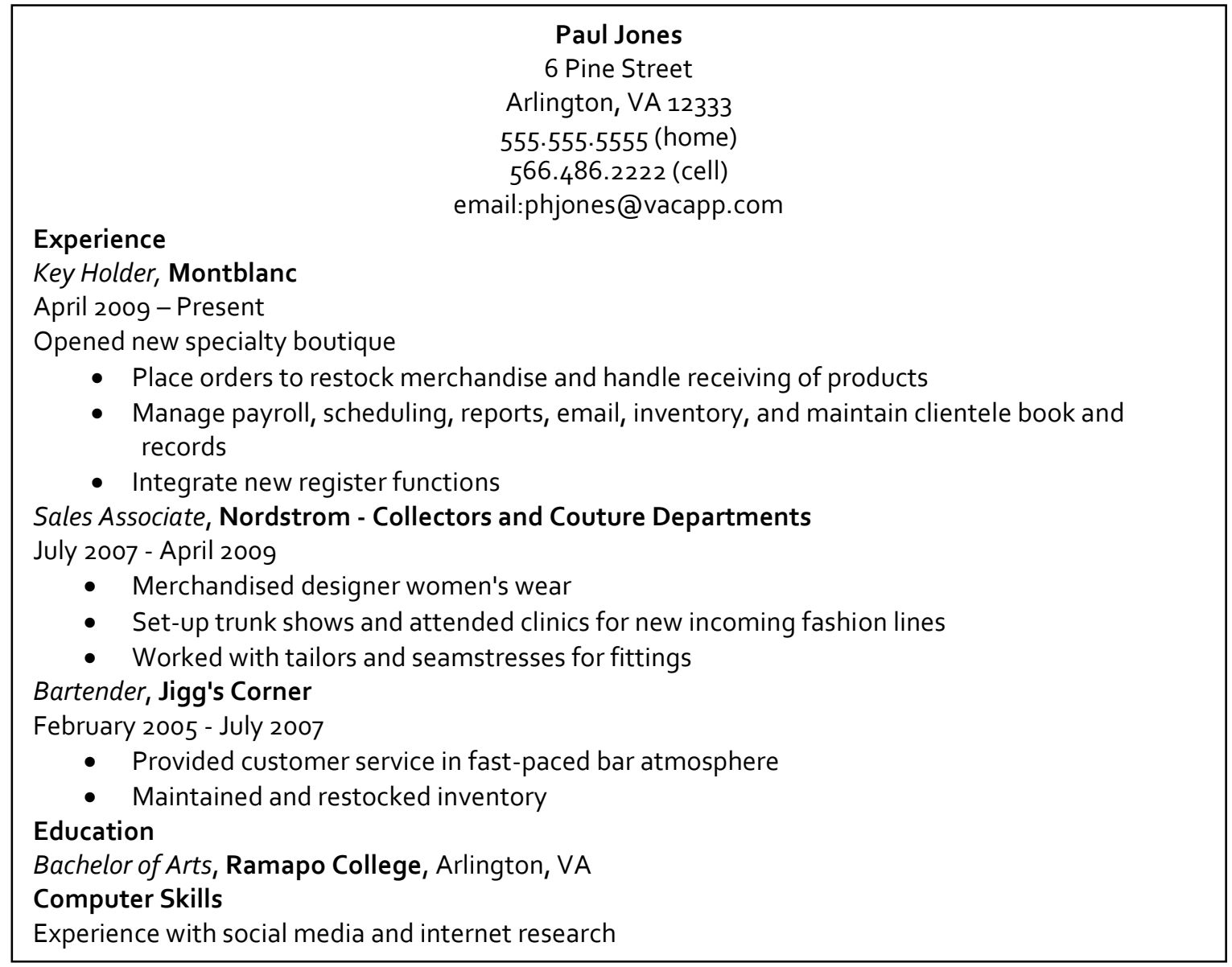

Fonte: http://jobsearch.about.com/library/samples/blretailresume.htm.

O résumé aqui exemplificado apresenta as realizações profissionais do candidato, postadas do item mais atual para os dados menos recentes. As questões alusivas às informações educacionais e habilidades são elencadas posteriormente.

No tocante à infraestrutura textual, observamos que o plano textual global (BRONCKART, 2012) dos résumés traz informações pessoais, resumo das qualificações, itens relacionados à experiência profissional e histórico acadêmico do candidato. Os dados listados são apresentados de forma clara e topicalizada, podendo conter informações extras que venham a somar em termos de qualificação profissional para a posição desejada. Visualizamos que o nome do candidato aparece em primeiro lugar (em negrito), seguido das informações pessoais, sendo que todos esses dados se encontram centralizados no texto. Em relação às qualificações, estas aparecem alinhadas à esquerda da página. Com o intuito de chamar a atenção do recrutador para as diferentes seções que compõem seu résumé, o agente produtor pode estilizar seu texto, fazendo uso de fontes diversas. A estrutura composicional não aparece em forma de um texto, com parágrafos e conectores 
interligando as partes a serem descritas. As informações são construídas em forma de itens e apresentam propriedades linguísticas particulares, como a ausência do pronome pessoal de terceira pessoa (he, em inglês). Uma vez que os dados referentes à vida profissional e acadêmica do sujeito aparecem enumerados no corpo do texto, podemos dizer que estes podem ser alocados no que Bronckart (2012) chama de outras formas de planificação, constituídas pelos scripts e esquematizações ${ }^{6}$.

\section{Metodologia de pesquisa}

O estudo é de natureza qualitativa, orientado pela metodologia da pesquisa-ação (BORTONI-RICARDO, 2008). Sobre os participantes, temos a presença de graduandos do curso de Letras (Português/Inglês) da Universidade Tecnológica Federal do Paraná (UTFPR), do Campus-Curitiba, da assistente de língua inglesa (ETA) e da professora pesquisadora. $O$ curso $^{7}$ de extensão Professional Writing Development (PWD) tem como princípio norteador as questões de mediação e interação com base em Vigotski (2001) e o estudo de gêneros (SCHNEUWLY; DOLZ, 2004). Dentre os instrumentos utilizados para geração de dados, trazemos excertos das interações orais ocorridas, transcritas para efeito de análise.

No que concerne à análise das inserções e interações orais, utilizamos as categorias dos modos de participação dos interlocutores na interação em eventos de comentários sobre textos em progresso proferidos por $\operatorname{Garcez}^{8}$ (2010, p. 93-97). Além dos critérios descritos pela autora, apresentamos uma expansão do rol de categorias a partir dos dados gerados durante as discussões construídas nas aulas, as quais estão agrupadas nos seguintes andaimes identificados ${ }^{9}$ : geração de ideias, socialização, negociação da interpretação e retomada.

\footnotetext{
${ }^{6}$ Segundo o autor, "Os scripts são próprios dos tipos da ordem do narrar, organizando os enunciados em uma ordem cronológica simples, que pretende reproduzir a ordem dos acontecimentos constitutivos da diegese. As esquematizações são próprias dos discursos teóricos e dos discursos interativos monologados, organizando as unidades de informação de acordo com procedimentos da lógica natural" (BRONCKART, 2012, p. 252).

${ }^{7}$ O curso PWD teve como objetivo geral desenvolver a compreensão e produção dos gêneros de texto biodata, résumé e cover letter, mas, para o propósito deste artigo, focalizamos somente o gênero résumé.

${ }^{8}$ Garcez (2010) apresenta os seguintes modos de participação dos interlocutores: releitura, categorias de objetivação, intersubjetivas, de intervenção no texto e de construção da reversibilidade. Para uma leitura mais aprofundada, ver obra intitulada A Escrita e o Outro (GARCEZ, 2010).

${ }_{9} \mathrm{Em}$ nossa tese de doutoramento, apresentamos um total de sete (7) andaimes. Para o propósito deste estudo, trazemos quatro (4) andaimes identificados, os quais se mostraram mais enfáticos no estudo do GP.
} 
Na categoria geração de ideias, temos as informações e ideias verbalizadas pelos participantes sobre o contexto de produção dos GP e sobre seus elementos constitutivos, em que diferentes posicionamentos são discutidos, refutados e/ou aceitos pelo grupo. Ainda nesta fase, trazemos como subitens a invocação à participação do outro, elicitado, de forma mais enfática, no início do curso pela ETA, por nossas inserções e, posteriormente, por todos os integrantes do curso. Temos, também, o item complementação de ideias, o qual se faz presente sempre que novos comentários sobre a temática profissional são proferidos nas trocas interativas.

A categoria socialização evidencia todas as experiências já vivenciadas pelos participantes, as quais têm a função de esclarecer, exemplificar e ajudar o outro a compreender melhor as instâncias em que determinado gênero é e/ou pode ser utilizado. Esta fase contempla, também, o compartilhamento de materiais adicionais e a realização de atividades instrucionais utilizadas para a construção do conhecimento. Juntamente a isso, a socialização de expressões e conteúdos relacionados tem a função de aprimorar o conhecimento linguístico da língua alvo.

A negociação da interpretação apresenta estreita relação com o conceito da ZDP (VIGOTSKI, 2002). Os seis (6) subitens que a compõem dizem respeito à construção do conhecimento a partir de um processo dialógico, de ajuda ao outro e da construção de conhecimentos científicos sobre os textos e contextos em que os referidos GP circulam. Todas as discussões aqui consideradas advém das trocas interacionais entre os participantes que, ao expor seus pontos de vista, têm a chance de sugerir diferentes formas de abordar o mesmo conteúdo, exemplificar questões relacionadas e explicar pontos obscuros. Além disso, tem-se o uso da LM como um instrumento mediador que vem esclarecer expressões ou situações não compreendidas pelos participantes.

Por fim, a categoria retomada, seja de falas e/ou de conteúdos relacionados à temática em estudo, intenciona enfatizar observações feitas no sentido de reciclar itens relevantes na constituição e nos contextos de uso dos GP.

Além da identificação dos andaimes mencionados, nossa análise também traz questões concernentes às capacidades de linguagem ${ }^{10}$ (SCHNEUWLY; DOLZ, 2004;

\footnotetext{
${ }^{10}$ As capacidades de linguagem (CL) são definidas como "aptidões requeridas do aprendiz para a produção de um gênero numa situação de interação determinada" (SCHNEUWLY; DOLZ, 2004, p. 54), fazendo com que os alunos, no momento das interações em sala de aula, se apropriem do gênero, ou gêneros em questão, a ponto de concebê-los como um instrumento mediador entre a pessoa e o agir social do sujeito por meio dele/deles.
} 
CRISTOVAO; STUTZ, 2011) e à formação da lista de constatação ${ }^{11}$ (BAIN, SCHNEUWLY, 1993; GONÇALVES, 2007) do gênero.

\section{Discussão dos dados}

O curso PWD proporcionou uma grande quantidade de dados advindos de discussões orais em torno da temática. Por meio da apresentação de fragmentos de textos (transcrição das vozes dos participantes), buscamos investigar em que medida as intervenções e discussões orais, mediadas pelas participações da professora pesquisadora, dos alunos, da ETA e dos diversos instrumentos mediadores utilizados (textos, atividades de leitura, exercícios de compreensão oral) atuaram na ZDP dos discentes.

Muitas foram as trocas interacionais observadas, as quais se deram nas discussões em pares e coletivas acerca do gênero. Com intuito de ilustrar os momentos de interação e mediação ocorridos, apresentamos algumas atividades realizadas em torno do gênero résumé. Iniciamos com uma atividade composta de orações falsas e verdadeiras, na qual são discutidas cinco (5) afirmações sobre o gênero. Essa atividade é uma adaptação de um exercício publicado no livro Resumé magic: trade secrets of a professional résumé writer (WHITCOMB, 2010).

Quadro 1- Atividade de discussão oral sobre résumés

When you are looking for a job one of the first goals is to equip yourself with the right tools. Based on what we have seen so far and on the ETA's own experiences and talks during our meetings, take the following quiz and see whether these statements are True or False (T/F).

a) Employers and candidates view résumés as having the same purpose. $\mathrm{T} / \mathrm{F}$

b) A résumé is all you need to get a job. T / F

c) A résumé is most effective when it precedes your interview with the hiring manager. T / F

d) Résumés are read thoroughly. $\mathrm{T} / \mathrm{F}$

e) You will need a résumé to land a job. T / F

Fonte: A autora. Adaptação da atividade elaborada por Whitcomb (2010).

As $C L$ são classificadas em capacidades de ação (CA), discursivas (CD), linguístico-discursivas (CLD) e de significação (CS), sendo a última uma proposição de Cristovão e Stutz (2011).

${ }^{11} \mathrm{~A}$ lista de constatação, também chamada de lista de controle, pode ser considerada como um instrumento regulador da aprendizagem discente, principalmente no que concerne ao processo da reescrita textual. Bain e Schneuwly (1993) apontam que "a lista de controle serve de baliza à aprendizagem. Ela ajuda os estudantes a antecipar ou a compreender os critérios de avaliação de seus textos, a fazer uma autocrítica de suas produções e, a partir deste momento, a estruturar e a modelar sua escrita no curso de sua realização" (BAIN; SCHNEUWLY, 1993, P. 68). 
A discussão sobre as questões foi pertinente para a construção das representações sobre as CS e CA, as quais fundamentaram as CD e CLD. Na sequência, temos parte do diálogo que ilustra as inserções dos participantes.

\section{Excerto 1}

206. A1: "A résumé is all you need to get a job". False!

207. ETA: False. Everybody agrees?

208. As: YES!

209. ETA: So what is necessary to have?

210. Ax: Qualifications/

211. A2: The information needs to be true.

212. Ay: Although some (+++) really famous people lie on the résumés. ((Ay talks about a famous guy who did a bunch of movies)). [...]

213. P: But remember it is like a combo! The résumé is one part.

214. ETA: Interview, cover letters/

215. A2: You have to show that you are really good. Impressive like the guy said!/

216: ETA: And even now ( $x x x$ in the US when you send them an email with your résumé and cover letter, you have to write a little something in the email. So it's also like a biodata, it has to be something personal in the first person. [...]

218. Ax: "A résumé is most effective when it precedes your interview with the hiring manager".

219. ETA: What do you think?

220. Ax. I don't know. I think it's a combo.

221. ETA: It's a combo. It's like a sandwich @@@.

222. Ay: It's true because if you send a résumé before the interview, the:: interviewer has a more clear vision of what he must look for in the interview.

223. ETA: Usually you have to have a résumé before $(+++)$ because they use the résumé to decide who gets to be interviewed.

(Aula 7- dia o3 de junho de 2014)

Neste pequeno excerto, temos a retomada de um dos elementos necessários na composição de um résumé, as qualificações do candidato (frase 210), alusivos às CD. Posteriormente, com o desenvolver do diálogo, percebemos que os participantes fazem alusão aos demais GP focalizados no curso (cover letter e biodata), bem como a outros gêneros relacionados à temática profissional, como o caso da entrevista e do e-mail, em que o candidato deve postar informações adicionais, complementando, de certa forma, o résumé enviado. Temos, aqui, uma amostra de um conjunto de gêneros alusivos à esfera profissional, os quais podem ser utilizados pelo candidato no momento em que participar de um processo de seleção de emprego.

As CS e CA também são contempladas quando A1, As, Ax e A2 (frases 206, 208, 210 e 211) apontam veementemente que o résumé não é o único gênero necessário para a colocação de uma pessoa no mundo do trabalho. Essa afirmação só é possível devido à experiência e/ou vivência de alguns alunos que já passaram por uma situação como esta, ou 
que tenham conhecimento sobre outros contextos em que diferentes gêneros foram utilizados para que eles pudessem agir em uma determinada situação de procura de um emprego. Entendemos que todos os gêneros por eles mencionados são representações de práticas sociais relacionadas com a realidade concreta que os cerca, os quais compartilham significados semelhantes percebidos nas novas circunstâncias que se apresentam.

A segunda atividade aqui ilustrada faz referência a um vídeo retirado da internet (http://www.youtube.com/watch?v=KbZ5 ar4Djo), intitulado Writing your Résumé. O vídeo apresenta asserções alusivas aos aspectos gramaticais e de ortografia (CLD), além do formato do gênero (CD). No diálogo que se estabelece (Excerto 2), há a retomada de questões previamente discutidas e a chance dos participantes realizarem uma atividade de compreensão oral (em inglês, listening). Além da socialização e compartilhamento de materiais extras que auxiliam no trabalho de reconhecimento do gênero, temos, ainda, a identificação dos critérios alusivos aos relatos de experiências vivenciadas, o incentivo às contribuições do outro e a socialização de expressões ou conteúdos relacionados, subitens referentes ao andaime socialização.

\section{Excerto 2}

43. A3: He says that he has seen thousands of résumés and people ask him why they should do it/ 44. P: That's why he::::: decided to make up this video, to help people/

45. ETA: He's probably seen a lot of BAD RÉSUMÉS!@@

46. P: Right. Next one Ax, can you read?

47. Ax: "b. According to him, what is the purpose of writing a résumé?" To expose yourself, $(x \times x)$ to give a better image of your career/

48. A3: To facilitate in the choosing.

49. P: Because, well, after the résumé, you can have like the interview.

50. ETA: Hopefully! [...]

52. A3: "c. He says a person can have more than one résumé. Why?" Because you have different jobs to apply for. They don't necessarily send the same résumé.

53. P: Depending on the company, or according to the company, you can include new information on it/

54. ETA: And you wanna make sure that every résumé is tailored to the specific job description, cause usually they're looking for specific words, so it's good to have a different aspect on it (++) for different jobs.[...]

56. ETA: [...] "d. Is it a good idea to mention your previous job?"[...]

57. A1: I understand that it's good to mention because the interviewer is going to know what you did, and what are you good at. And it's more related to the next question.

58. P: Yeah, and to talk a little bit about you professional ah::: history. If I can say that?

59. ETA: Hum, hum. Yes, you can say that, yes!

60. A3: The expression JOB HOPPER. Is it a very common expression in English?

61. ETA: Yeah, I've heard it before. But there's other names for it, but it's, yes.

62. P: Do we have an expression for this in Portuguese?

63. A1: I can't think of any::: [...]

65. A1: It's someone who doesn't keep jobs. [...] 
70. P: And here we have the definitions of a résumé which is a French word, and a job hopper meaning "someone who works briefly in one position after another rather than staying at any one job or organization long-term" [...]

(Aula 8- dia 10 de junho de 2014)

Nas orações 52, 53 e 54, observamos o andaime retomada de falas e conteúdos, cujas informações haviam sido pontuadas anteriormente com a menção de que um novo résumé pode ser fabricado a partir das exigências do empregador. Nas frases 58 e 59, temos a negociação da interpretação e o incentivo à contribuição do outro, por meio dos itens conversacionais Hum, hum e da palavra yes. Tais itens aparecem nos apontamentos da professora pesquisadora e da ETA ao confirmarem o comentário proferido por A1 (frase 57) sobre a relevância da menção das realizações do candidato em empregos anteriores, relatando, mesmo que de forma breve, seu histórico profissional.

Ainda no fragmento selecionado, apontamos a frase 6o, em que $A_{3}$ indaga a ETA sobre a utilização da expressão job hopper no contexto americano. Observamos o andaime socialização de expressões e conteúdos relacionados aos GP e a explicação de vocabulário desconhecido na língua alvo, com a leitura (frase 70) da definição do termo. Temos, aqui, um exemplo do que Vigotski (2008) chama de transferência de um significado já apropriado em um determinado sistema linguístico para outro que ainda se encontra em processo de desenvolvimento. Há, portanto, o estabelecimento de uma interação entre duas línguas, em que a utilização da LM se apresenta como fundamental para a aprendizagem da LE.

No que tange à intenção de trabalhar com a compreensão oral via um vídeo utilizado em um contexto americano de produção textual, defendemos que textos referentes às novas mídias podem ser inseridos no contexto de sala de aula, pois auxiliam e facilitam o trabalho do professor com relação à organização de atividades voltadas a uma temática específica, além de apontar para diferentes visões de textos e contextos, aproximando realidades distantes do cenário educacional.

Sobre a construção da lista de constatação (BAIN; SCHNEUWLY, 1993; GONÇALVES, 2007), trazemos o Excerto 3, em que o grupo decide sobre os elementos que devem ser focalizados na escrita de um résumé. A conversa tem início com a comparação do GP com o CV Lattes.

\section{Excerto 3}

1. P: If you compare the two, the two genres $(++)$ our Lattes and these résumés.

2. Ax: $(x x x)$ In the résumés there's like $(x x x)$ of computer stuff $(x x x)[\ldots]$ 
3. P: OK. So, skills is one element that they usually have.

4. Ax: Yes, the computer ones ( $x x x)$

5. P: The computer ones. Yeah, because in the Lattes we don't have that!

6. Ax: $(x x x)$

7. P: So here, in terms of skills, it is more related to languages. Yeah? (+++) [...]

8. Ay: You have a description of his employment history which you didn't have in the CV.

9. P: Ah, ah. Could you repeat Ay? A description of $(++)$

10. Ay: The employment history. (++++)

11. P: Oh yeah, employment history. $(++++++)$

11. ETA: That's one thing that I really like about résumés is you get to, you have to say, oh I was, I did this, it's like you have the title and you also get to present yourself and say I accomplish this (xxx). You have the chance to explain a little bit more.

12. P: Yeah, in the CV Lattes we have, for example, I worked at Cultura Inglesa, or JUST Cultura Inglesa. And they know NOTHING about my job. So I can't say anything about my job, what I did in there, but in the ré:su:mé [you can]! [...]

15. P: You know what we have in Brazil now, if we take a contest, for example, we have a memorial. Have you ever heard of this? So you have to make your OWN MEMORIAL which is you talk about yourself, and you include things in there. [...]

18. Ay: It's like a biodata!

19. P: It's a kind of biodata, but it's LONGER. It's much longer. [...] So, how can we (++) put this? $(++++)$ So you can talk about your history, the history you had/

20. ETA: A DESCRIPTION OF THE POSITION.

21. P: A description of the position. Ah, ah. Good!

22. A2: You can give a detail or details about what you have done::

23. ETA: And one thing they always encourage us to $(x x x)$ what you have accomplished or done in that position. (++++)

26. P: Did you have to do this ETA to come to Brazil? When you decided to come, when you applied for it? (+++) You don't remember?

27. ETA: I DO! I was trying to remember. I know I did talk about work experience; it was kind of like a CV Lattes. WORK EXPERIENCE and you had the chance to write it. (XXX) It was an online application. We had to talk about all our work experience. [...]

29: ETA: I think it's some of the kind of QUALIFICATION accomplishments.

30. P: OK. Is it shorter than the CV Lattes? ((In comparison to a résumé))

31: As: Hum, hum. Yeah

32. Ay: You have more freedom like how you present that. [...]

33. A2: I think, I don't know, I think that in Brazil we don't have a ( $x x x)$ résumé. You follow a more or less (xxx) but it's not the main thing, obrigatório, I don't know/

34. As: [Mandatory] Compulsory.

35: $A_{3}:$ I think $[\ldots]$ in the résumé you have to do things more specific for the job you want.

36: P/ETA: Hum, hum.

37: P: Very Good. That's THE POINT!

(Aula 7- dia o3 de junho de 2014)

Por meio do diálogo, vemos que a negociação passa a circunscrever-se aos elementos característicos do gênero (CD). À medida que os comentários dos participantes se tornam mais enfáticos, há a criação da lista de constatação organizada pelo grupo. Focalizando as questões interacionais, o excerto ilustra perguntas do tipo professor-alunos, em que a forma de interação entre os participantes acontece mais naturalmente, sem que 
haja a necessidade de elicitar a participação de um determinado aluno. Quando se evoca uma participação mais específica, isto se deve em razão da necessidade de repetição de palavras/frases no sentido de enfatizar itens/explicações mencionadas, como é o caso das frases 9 , 10 e 11, com o objetivo de que o aluno seja ouvido por todos e sua asserção seja compreendida e aceita no grupo.

Ao longo da conversa, observamos uma intensa participação dos interlocutores, com a verbalização de contextos previamente vivenciados por alguns dos participantes, como na frase 11, em que a ETA aponta uma vantagem do GP résumé, ao mencionar que o escritor pode explicar melhor sobre as realizações profissionais anteriormente vividas. Sobre o seu apontamento, vemos que a ETA atua como um par mais experiente (VIGOTSKI, 2002) no que tange ao uso do GP, uma vez que este é um gênero de grande veiculação no cenário profissional americano. Temos, ainda, o comentário de $\mathrm{P}$ na frase 12, o qual aponta para semelhanças e/ou diferenças existentes entre os contextos de produção (CA) e características dos GP (CD) em questão.

A despeito dos demais gêneros de texto trazidos para o contexto de discussão, há a menção do gênero memorial (frase 15), utilizado em concursos públicos voltados para a área da educação, o qual é comparado a uma biodata (frase 18). Tal comentário é confirmado por $P$ (frase 19), a qual atenta para uma extensão de texto maior para o gênero memorial.

No diálogo que se instaura (frases 20 a 29), a estrutura composicional (CD) do GP começa a ser delineada pelos participantes e tem-se a descrição das posições ocupadas pelo candidato em empregos anteriores, suas qualificações e, principalmente, especificidades relacionadas a cada uma das qualificações mencionadas. Tais itens têm como objetivo chamar a atenção do recrutador para que o candidato seja, em uma etapa posterior, selecionado para uma entrevista de emprego. Demonstrando envolvimento dos participantes sobre a temática, as categorias subjetivas (GARCEZ, 2010) existentes no diálogo emergem por meio dos itens conversacionais yeah, hum hum, correspondentes à negociação da interpretação e da verbalização de julgamento de valor, com as expressões good, very good, that's the point.

Os comentários finais (frases 33 a 37) apontam para a função da organização do conteúdo ( $\left.{ }_{3} C D\right)$ do GP, verbalizando, de forma direta, o objetivo de se escrever um résumé, evidenciado na frase 35 de $A_{3}$. Ao trazer como marca linguística a expressão 'I think', esta objetiva conferir largas parcelas de texto sintetizadas em uma só frase, a qual é, na 
sequência (frases 36 e 37), confirmada pelos demais participantes. Visualizamos, portanto, que as CA, CS e CD (BRONCKART, 2007; CRISTOVÃO; STUTZ, 2011) se encontram articuladas com o objetivo de informar o leitor sobre as realizações profissionais do escritor.

Com o objetivo de sintetizar e reciclar as informações anteriormente pontuadas, os participantes novamente enumeram os itens elencados como relevantes em um résumé. Essa retomada de elementos estruturais emerge a partir da pergunta proferida pela ETA.

\section{Excerto 4}

108. ETA: So what are the parts that comprise a résumé? [...] What did the samples have? They have the NAME, ADDRESS, PHONE NUMBERS, EMAIL ADDRESSES. Anything else that you can:::: [...] So after the personal information, what do you think we should a:::dd [...]

109. $A_{3}$ : There is one here (+++) that has the GOAL $(x x x)$.

110. P: Yeah, in some, 3 or 4 samples we had a goal.

111. ETA: Or the OBJECTIVE. [...] We can use the two words. [...]

113. A1: WORK EXPERIENCE.

115. ETA: It depends on what $(+++)$ I would put EDUCATION first.

116. P: In the Lattes I think we have education first, we have the DEGREE, and after that we have work experience. What do you think, people?

117. A1. For me it would be experience because I would say I work for the Air Force. People would look at me differently.

119. A3: I have put my experiences first and the, the other courses I have done.

120. P: OK, after these two items, what do you think we could use? [...]

121. Ax1: I think in our case we could use the languages.

122. A3: There is one thing that we haven't talked about. Here in Brazil we use, at the end, some REFERENCES. Can we use that?

123. A1: Like in the video, the guys says that we can put it.

124. ETA: Sometimes they ask for references, but not on the résumé. [...]

125. P: Maybe in a cover letter. In the Lattes we never use that!

126. A3: Depending on the job they would ask for 2 or 3 references. [...]

127. P: When I tried [...] my mestrado, [...], I had to present 2 reference letters. [...]

128. ETA: I had to do too for this program. ((The Fulbright Program))

129. P: OK, after the language skills, [...]

130. As: SOFTWARE SKILLS.

131. ETA: CONTINUING EDUCATION, or OTHER COURSES, or CERTIFICATES. [...]

132. A1: We could use OTHER CERTIFICATIONS 'cause [...] we don't have a name for it.

133. ETA: Anything else? Memberships? [...]

134. A3: AFFILIATIONS.

135. P: Yeah, it's the last part in our Lattes. Like Associação Brasileira de Linguística Aplicada. So, I'm a member, [...]. Do you think further information would be nice to use? [...]

136. ETA: I think we could have, you can put MEMBERSHIPS. [...]

137. A1: In my case, I would put work experience like I work for the Air Force, and in other certifications I would put everything related to other courses. [...]

(Aula 8 - dia 10 de junho de 2014)

Ao indagar os participantes sobre as partes que compõem o GP (CD), a ETA menciona alguns de seus elementos principais (nome; endereço; números de telefone e 
endereço eletrônico). Com esse comentário, há um aumento da participação dos demais membros, em que os interlocutores adicionam outras informações responsáveis por sua estruturação.

Temos, assim, a menção do objetivo, que aparece em um dos textos de referência analisados (frase 109 de $A_{3}$ ), a experiência profissional (frase 113) e o item educação (frase 115). Além disso, os elementos habilidades, outros cursos realizados e afiliações também são lembrados como relevantes neste GP. As frases 117, 119 e 121 focalizam, mais especificamente, os pontos de discussão para a realidade dos participantes. A3 (frase 122) chama a atenção para a questão da referência (quando uma pessoa da área é solicitada a fornecer informações adicionais sobre o candidato). Por já ter experiência com a escrita do GP, a ETA sugere que tal item não seja utilizado, posicionamento confirmado pela professora pesquisadora (frase 125) ao enfatizar que a plataforma Lattes também não permite que essa informação seja postada.

O diálogo apresentado abre a possibilidade para a construção de um Mapa Mental, o qual tem como objetivo ilustrar o caráter dialógico da análise efetuada em torno do GP.

Figura 2 - Mapa mental: lista de constatação do GP résumé

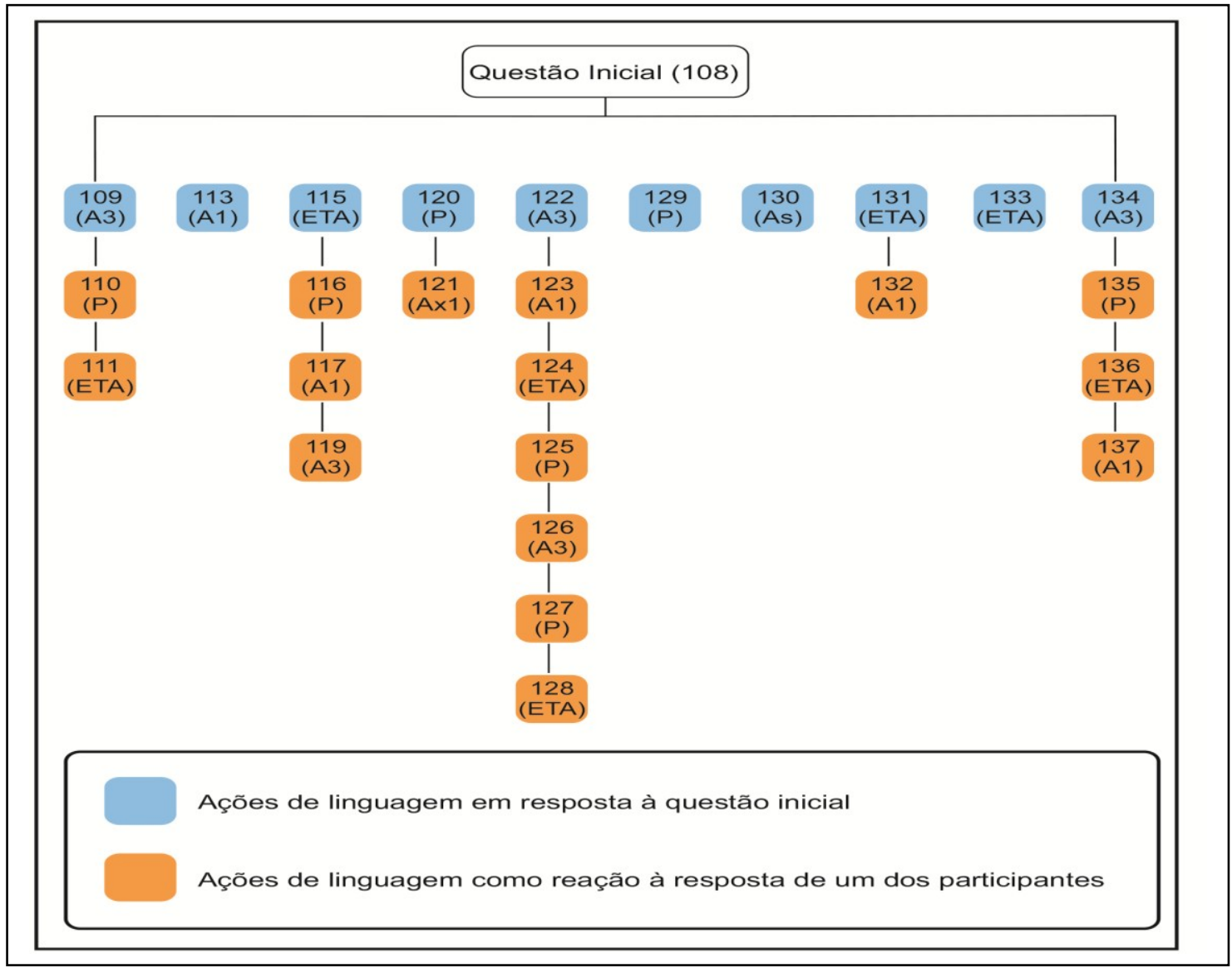

Fonte: A autora, com base em Drogui (2014). 
A organização do mapa mental representa a materialização das trocas interacionais ocorridas ao longo do curso, as quais são responsáveis por desencadear vários processos de aprendizagem. A ação de linguagem proferida pela ETA na frase 108 (So what are the parts that comprise a résumé? [...] What did the samples have? They have the NAME, ADDRESS, PHONE NUMBERS, EMAIL ADDRESSES. Anything else that you can:::: [...] So after the personal information, what do you think we should a:::dd [...]) faz com que haja um desencadeamento de novas inserções pontuadas pelos participantes até que se chegue a um concenso sobre os elementos necessários na composição do GP.

As diferentes inserções sugeridas atuam na ZDP (VIGOTSKI, 2002) dos participantes, fazendo-os compreender as variáveis que compõem o gênero. Assim, tomando o GP résume como um instrumento psicológico de mediação, observamos que há uma ampliação dos diferentes conhecimentos que, ao serem compartilhados, formulados e reformulados com o outro, incitam o desenvolvimento mútuo de novos saberes.

\section{Considerações Finais}

As trocas interacionais e os vários instrumentos mediadores utilizados ao longo do curso proporcionaram um ambiente colaborativo na aprendizagem do gênero de texto desconhecido pela maioria dos participantes. Em relação aos papéis e posicionamentos assumidos pelos participantes, é possível afirmar que houve mais autonomia e forte participação dos discentes nas inserções realizadas durante as discussões, proporcionando o desenvolvimento de suas potencialidades, que, de acordo com a perspectiva vigotskiana, podem ser transformadas a partir das trocas interacionais com o outro.

Com relação aos andaimes identificados, a complementação de ideias foi um constante no curso e pode ser observado nos excertos aqui analisados, pois a partir de um questionamento, dúvida ou inserção a respeito de um item relacionado à temática, aspectos de ordem contextual ou linguística do gênero résumé foram complementados.

Sobre as atividades realizadas, ressaltamos que a atividade sobre questões falsas verdadeiras foi a que mais suscitou a partipação do grupo em termos de uma negociação ampla da interpretação sobre a temática. Essa negociação se deu por meio da inserção de informações provenientes de outras fontes de dados, mobilizando conhecimentos e histórias de vida de cada um dos participantes, ao tomarem uma posição sobre a relação 
texto-contexto e ao relacionarem aspectos macro do gênero com a realidade da qual eles fazem parte.

\section{Referências bibliográficas}

BAIN, D.; SCHNEUWLY, B. Por une evaluation formative intégrée dans la pédagogie du français : de la nécessité et de l'utilité de modèles de référence. In: PERRENOUD, P.; BAIN, D.; $A L L A L, L$. Évaluation formative et didactique du français. Lausanne: Delauchaux et Niestlé, 1993, p. 51-79. Disponível em: file:///C:/Users/Ana/Downloads/unige_34345_attachmento1.pdf. Acesso em: 17 mar. 2015.

BORTONI-RICARDO, S. M. O professor pesquisador: introdução à pesquisa qualitativa. São Paulo: Parábola, 2008.

BRONCKART, J. P. Atividade de linguagem, textos e discursos: por um interacionismo sociodiscursivo. $2^{\text {a }}$ ed. Trad. Anna Rachel Machado, Péricles Cunha. São Paulo: EDUC, 2012.

CAZDEN, C. B. Classroom discourse: the language of teaching and learning. Porthmouth, N.H.: Heinemann, 1988.

CRISTOVAO, V. L. L. ; STUTZ, L. Sequências didáticas: semelhanças e especificidades no contexto francófono como L1 e no contexto brasileiro como LE. In: SZUNDY, P. T. C.; ARAÚJO, J. C.; NICOLAIDES, C. S.; SILVA, K. A. (Orgs.). Linguística aplicada e sociedade: ensino e aprendizagem de línguas no contexto brasileiro. Campinas, SP: Pontes Editores, 2011, p. 17-39.

DONATO, N. Collective scaffolding in second language learning. In: LANTOLF, J. P.; APPEL, G. (Orgs.). Vygotskian approaches to second language research. Norwood: Ablex, 1994, p. $35-56$.

DROGUI, A. P. Interação no meio virtual e ensino de línguas: análise de uma proposta para a formação de professores de língua espanhola. 2014. Dissertação (Mestrado em Estudos da Linguagem). Universidade Estadual de Londrina, Londrina, 2014. 141 f. : il.

FIGUEIREDO, J. F. O. A aprendizagem colaborativa: foco no processo de correção dialogada. In: LEFFA, V. J. (Org.). A interação na aprendizagem de línguas. Pelotas: EDUCAT, 2003, p. 125-157.

FRIEDRICH, J. Lev Vigotski mediação, aprendizagem e desenvolvimento: uma leitura filosófica e epistemológica. São Paulo: Mercado das Letras, 2012.

GARCEZ, L. H. C. A escrita e o outro: os modos de participação na construção do texto. Brasília: Editora UnB, 2010. 
GONÇALVES, A. V. Gêneros textuais e reescrita: uma proposta de intervenção interativa. 2007. Tese (Doutorado em Ensino/Aprendizagem de Línguas). Universidade Estadual Paulista "Júlio de Mesquita Filho", Araraquara, 2007. 344f.: il.

LEFFA, V. J. (Org.). A interação na aprendizagem de línguas. Pelotas: EDUCAT, 2003, p. $125-157$.

MOITA LOPES, L. P. Uma linguística aplicada mestiça e ideológica: interrogando o campo como linguista aplicado. In: MOITA LOPES, L. P. (Org.) Por uma linguística aplicada INdisciplinar. São Paulo: Parábola, 2006, p.13-44.

PAIVA, V. L. M. O. Feedback em ambiente virtual. In: LEFFA, V. J. (Org.). A interação na aprendizagem de línguas. Pelotas: EDUCAT, 2003, p. 219-254.

REGO, T. C. Vygotski: uma perspectiva histórico-cultural da educação. Petrópolis, RJ: Vozes, 1995.

SCHNEUWLY, B.; DOLZ, J. (org.). Gêneros orais e escritos na escola. São Paulo: Mercado de Letras, 2004.

VIGOTSKI, L. S. A formação social da mente: o desenvolvimento dos processos psicológicos superiores. São Paulo: Martins Fontes, 2002.

Pensamento e linguagem. 4 ed. São Paulo: Martins Fontes, 2008.

WHITCOMB, S. B. Résumé magic: trade secrets of a professional résumé writer. Fourth Edition. JIST Publishing, 2010.

WOOD, D.; BRUNER, J. S.; ROSS, G. The role of tutoring in problem solving. Journal of Child Psychology and Psychiatry and Applied Disciplines. v. 17, n. 2, 1976, p. 89-100.

\footnotetext{
i Ana Valéria Bisetto Bork, Profa.

Universidade Tecnológica Federal do Paraná - Campus Curitiba

Doutora em Estudos da Linguagem. É professora titular de língua inglesa do Departamento Acadêmico de Línguas Estrangeiras Modernas (DALEM) da UTFPR - Campus Curitiba. Seus interesses de investigação englobam o ensino e aprendizagem de língua inglesa, estudo de gêneros e formação inicial de professores.

valeriabbork@hotmail.com
} 